This is in the post peer review accepted manuscript of:

Kamienski C, Soininen J-P, Taumberger M, Fernandes S, Toscano A, Salmon Cinotti T, Filev Maia R, Torre Neto A, "SWAMP: an loT-based Smart Water Management Platform for Precision Irrigation in Agriculture", 2018 Global Internet of Things Summit (GloTS), Bilbao, 2018, pp. 1-6.

DOI: $10.1109 /$ GIOTS.2018.8534541

The published version is available online at: https://doi.org/10.1109/GIOTS.2018.8534541

(C) 2018 IEEE. Personal use of this material is permitted. Permission from IEEE must be obtained for all other uses, in any current or future media, including reprinting/republishing this material for advertising or promotional purposes, creating new collective works, for resale or redistribution to servers or lists, or reuse of any copyrighted component of this work in other works 


\title{
SWAMP: an IoT-based Smart Water Management Platform for Precision Irrigation in Agriculture
}

\author{
Carlos Kamienski ${ }^{1}$, Juha-Pekka Soininen ${ }^{2}$, Markus Taumberger ${ }^{2}$, Stenio Fernandes ${ }^{3}$, \\ Attilio Toscano ${ }^{4}$, Tullio Salmon Cinotti ${ }^{4}$, Rodrigo Filev Maia ${ }^{5}$, Andre Torre Neto ${ }^{6}$ \\ cak@ufabc.edu.br,Juha-Pekka.Soininen@vtt.fi,Markus.Taumberger@vtt.fi,stenio@cin.ufpe.br, \\ attilio.toscano@unibo.it,tullio.salmoncinotti@unibo.it,rfilev@fei.edu.br,andre.torre@embrapa.br \\ ${ }^{1}$ Federal University of the ABC, Santo André/Brazil \\ ${ }^{2}$ VTT Technical Research Centre of Finland, Oulu/Finland \\ ${ }^{3}$ Federal University of Pernambuco, Recife/Brazil \\ ${ }^{4}$ University of Bologna, Bologna/Italy \\ ${ }^{5}$ Centro Universitário da FEI, São Bernardo do Campo/Brazil \\ ${ }^{6}$ Brazilian Agricultural Research Corporation (EMBRAPA), São Carlos/Brazil
}

\begin{abstract}
Irrigation for agriculture is the biggest consumer of freshwater in the world, which makes a case for the intensive use of technology to optimize the use of water, reduce the consumption of energy and improve the quality of crops. While the Internet of Things (IoT) and other associated technologies are the natural choice for smart water management applications, their appropriateness is still to be proven in real settings with the deployment of on-site pilots. Also, IoT-based application development platforms should be generic enough to be adapted to different crops, climates, and countries. The SWAMP project develops IoT based methods and approaches for smart water management in precision irrigation domain and pilots them in Italy, Spain, and Brazil. In this paper, we present the SWAMP view, architecture, pilots and the scenario-based development process adopted in the project.
\end{abstract}

Keywords - Internet of Things, Smart Water Management, Precision Irrigation

\section{INTRODUCTION}

Water is vital for ensuring food security to the world's population, and agriculture is by far the biggest consumer amounting to $70 \%$ of freshwater [7]. The leading causes of water wastage are leakages in distribution and irrigation systems, as well as, the field application methods, which result in wastage and the cultivation of crops. The most common irrigation technique, surface irrigation, is also the least precise one, which wastes a high percentage of the water by wetting areas where no plants can benefit from it. On the other hand, localized irrigation can use water more efficiently and effectively, avoiding both under-irrigation and over-irrigation. However, in an attempt to avoid loss of productivity by underirrigation, farmers feed more water than is needed and as a result not only productivity is challenged but also water is wasted. Therefore, technology should be developed and deployed for sensing the level of water needed by the plantation and for flowing the water in the proper amount to places where and when it is needed.

The Internet of Things (IoT) [1] and other related technologies can be used for that purpose, but it faces several challenges. Firstly, Software Development for IoT-based smart applications is today not automatized and demands much effort. Secondly, advanced platforms are needed, that can automate part of the process and integrate different technologies and components for dealing with a multitude of requirements. Thirdly, the integration of heterogeneous and advanced sensors, particularly flying sensors (i.e., drones), may provide precision in the water supply for irrigation in agriculture. Fourthly, the use of a Software Platform together with different technologies such as IoT, big data analytics, cloud computing, fog computing [3] and drones, for the deployment of pilot applications for smart water management. Fifthly, new business models for using IoT in smart water management settings are necessary. Moreover, finally, components must be interoperable, flexible and adaptable to be replicable to different locations, crops, and contexts.

The primary objective of the $\mathrm{SWAMP}^{1}$ project is to develop IoT based methods and approaches for smart water management in precision irrigation domain and to pilot the approaches in four places, two pilots in Europe (Italy and Spain) and two pilots in Brazil. Also, we aim at improving precision irrigation by increasing the awareness of the condition of the crop, by monitoring the field based on crop status (size, growing phase) and environment (e.g., weather forecast) and to adjust the irrigation prescription map accordingly. The smart water management pilots aim at guaranteeing that technological components are flexible enough to adapt to different contexts and to be replicable in different locations and settings. The same underlying SWAMP platform can be customized to different pilots considering different countries, climate, soil, and crops.

The SWAMP layered architecture considers three types of services to ensure its replication and adaptability to different crops and locations. Entirely replicable services deal with IoT services, virtual entities and storage services, and data analytics and machine learning, respectively. Fully customizable services deal with water data management issues that specialize generic analytic services into particular techniques for different types of irrigation and water distribution. Those services may require being customized whenever a new pilot is designed, developed and deployed. Finally, application specific services require higher development effort since they serve particular farms. The architecture may be implemented in a range of deployment configurations involving the use of smart algorithms and analytics in the cloud, fog-based smart decisions located on the farm premises and possibly mobile fog

\footnotetext{
${ }^{1}$ swamp-project.org
} 
nodes acting in the field (e.g., drones or in the central pivot irrigation mechanisms). In this paper, we present the SWAMP view of water management, the SWAMP architecture, the four SWAMP pilots and a scenario-oriented development process that consider both the crop needs and technological solutions.

In the remainder of this paper, section II introduces concepts and related work. Section III introduces SWAMP concepts and methodology, while section IV presents the four pilots. Section V presents scenarios for deploying the pilots, and finally, section VI draws conclusions and future work.

\section{IOT IN PRECISION IRRIGATION FOR AGRICULTURE}

The Internet of Things is expected to have a decisive influence in the automation of smart water management applications. However, the development of IoT-enabled applications still takes tremendous efforts due to the lack of proper tools [8]. This scenario opens new opportunities and challenges in the specification, implementation, deployment, and evaluation of software development platforms for smart water management.

The SWAMP project is being built upon existing efforts, generic platforms, architectures and research results [13]. The IoT-A project developed the IoT Reference Architecture for the generation of compliant IoT concrete architectures, focusing on using the current state of the art, rather than applying a clean slate approach [2]. FIWARE evolved from an EU-funded initiative for cloud services and now includes a platform with a set of Generic Enablers for developing smart applications [12], such as in the precision irrigation domain for agriculture [10].

When it comes to providing advanced features to the management of natural resources, such as water, there are some isolated initiatives not necessarily connected to the existing platforms and architectures. For example, the FIGARO project aims at increasing water productivity and improving irrigation practices through the development of a precision irrigation management platform, but not directly involving IoT [6]. Also, Popović et al. [11] present a case study of the specially designed and currently limited IoTenabled platform for collecting data in precision agriculture and ecological monitoring domains. Agri-IoT [9] is a theoretical IoT-based framework for data analytics and realtime processing for smart farming that shares some similarities with SWAMP. However, so far no real deployments have been reported, making it difficult to compare with SWAMP.

In the last years, much has been said about the prospective uses for IoT combined with cloud-based services and big data analytics. Now, the time has come to conduct experiments in real settings. In Europe, there is a current concern to understand the challenges and compelling impacts of IoT in large-scale pilots for smart agriculture. Brewster et al. discuss the deployment of those large-scale pilots for IoT in agriculture and describe technologies and solutions that might be present in some agrifood domains, such as dairy, fruit, arable crops and meat \& vegetable supply chain [5]. Also, the European project IoF2020 aims at fostering the adoption of IoT in large-scale pilots in the farming and food domain ${ }^{2}$.

${ }^{2}$ iof2020.eu
Current existing solutions are mostly theoretical with limited proof of concept experiences. They are either too generic or too specific and do not explicitly address easy system development and deployment for facilitating replicability of scenarios and streamlining the deployment of new pilots. This is where SWAMP comes to play.

\section{CONCEPT AND METHODOLOGY}

The SWAMP project is developing a high-precision smart irrigation system concept for agriculture depicted in Fig. 1. The fundamental idea is to enable optimizations of irrigation, water distribution, and consumption based on a holistic analysis that collects information from all aspects of the system including even the natural water cycle and the cumulated knowledge related to growing particular plants. It results in savings to all parties as it guarantees the availability of water in situations where water supply is limited and also prevents over- and under-irrigation.

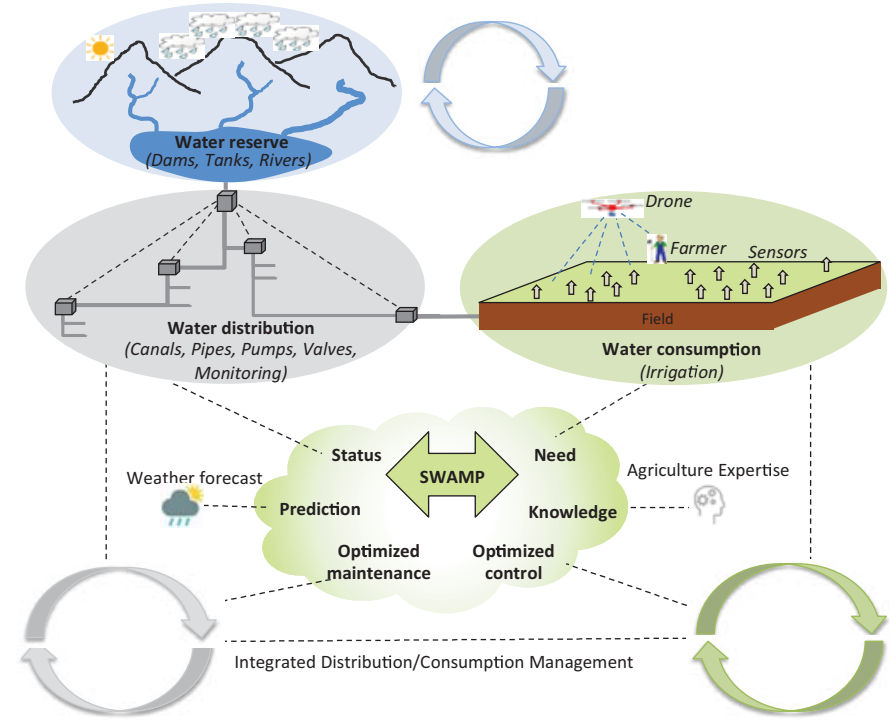

Fig. 1. Precision irrigation based on smart water management

As depicted in Fig. 1 we identify three broad phases in a water management system for agriculture:

- W1: Water reserve: water reserves coming from different sources such as rivers, lakes, dams, and aquifers, which follow the natural water cycle.

- W2: Water distribution: water is transported from W1 to the final usage place (W3) through a network of canals, pipes, pumps, valves, and gates. Water distribution may assume different configurations depending on the region or country. In some places, water resources are carefully used and controlled by a central authority.

- W3: Water consumption: in agriculture, one of the critical uses of water is irrigation, which can be performed by different techniques. Irrigation (W3) is the principal objective of the SWAMP project.

The SWAMP platform provides mechanisms for data acquisition from sensors, making decisions using a variety of different techniques and changing system behavior by sending commands to both automatic and human actuators. For Water Consumption (W3) SWAMP will provide real-time responses 
for adapting irrigation as crop conditions change. On the other hand, as changes in water distribution are performed in a different timescale, the management loop for W2 will be longer. W2 and W3 management systems are integrated, as water usage triggers water distribution. The SWAMP Architecture is divided into five layers, as depicted in Fig. 2.

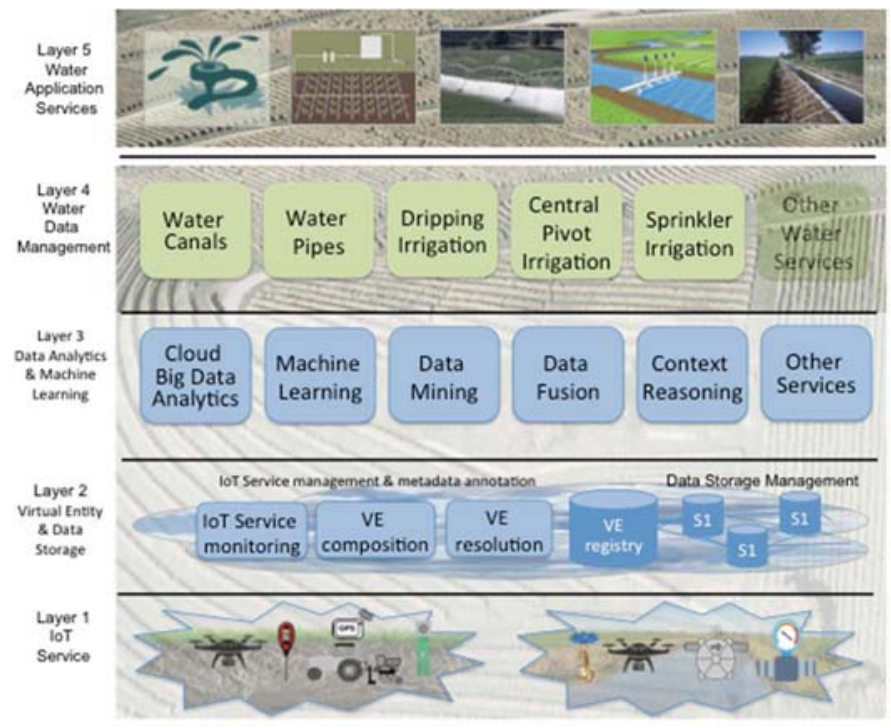

Fig. 2. SWAMP Architecture

- Layer 1: IoT Services: A variety of sensor and actuator technologies to acquire soil (e.g., moisture and temperature), plant (e.g., vegetation index, canopy temperature) and weather (e.g., air temperature and relative humidity, precipitation, solar radiation, wind speed, and direction) will be tested during the project.

- Layer 2: Virtual Entity and Data Storage: IoT Service descriptions are annotated with contextual metadata about the physical environment to create Virtual Entity (VE) representations of physical entities. Distributed databases composed of cloud and fog nodes work together for dealing with a massive amount of data coming from sensors and make them available to the upper layers.

- Layer 3: Data Analytics: provides different components for context-aware management supported by cloud-based big data analytics techniques. SWAMP will utilize existing models and algorithms to realize the Layer 3, which may vary for different crops and pilots.

- Layer 4: Water Data Management: builds application related middleware management services on top of the generic data processing services provided by Layer 3 . This layer decouples the irrigation-related business rules from applications providing an API for Layer 5 with a variety of algorithms, models, and mechanisms for facilitating and streamlining the development and deployment of new pilots.

- Layer 5: Water Application Services: A multitude of data that is sensed, acquired, stored, and processed is transformed into services that make sense to farmers. SWAMP initially will consider two types of water application services, water distribution services, and water usage services (i.e., irrigation).
A fundamental idea in SWAMP is to facilitate the replication of water management systems built on top of its platform with minimum redesign and redevelopment. Different layers of the architecture have components that are more generic and thus less difficult to be ported to other pilots, whereas others are more application-specific and thus require new development efforts whenever a new pilot is conceived. When it comes to the generality/specificity scale, the SWAMP architecture provides three categories of components: a) Fully Replicable Services: Layers 1, 2 and 3 of the architecture are generic enough to allow them to be replicable in different settings. Sensors, actuators and associated IoT Services vary according to the specific application domain; b) Fully Customizable Services: Layer 4 provides services that are closer to the final application and therefore must be aware of a level of detail that may vary for different techniques and models of water distribution and irrigation. Those services may require being customized for every new pilot; c) Application Specific Services: Services in Layer 5 address particularities of pilots. Given that parameters vary considerably for different countries, regulations, climate conditions, crops and soils, those services usually require more effort to be customized.

\section{SWAMP PILOTS}

\section{A. Pilot: Smart Water Distribution (Italy - CBEC)}

The Consorzio di Bonifica Emilia Centrale (CBEC) is a reclamation consortium of the Emilia-Romagna Region in Northern Italy, responsible for the irrigation and water drainage of an area of nearly $3,130 \mathrm{~km}^{2}$ where most water required for irrigation is withdrawn from the Po river (Fig. 3). The water is distributed to the farms by an intricate irrigation infrastructure composed of more than $3580 \mathrm{~km}$ of canals, more than 200 small streams, six draining plants, and 72 pump stations with the capacity of 416 cubic meters per second.

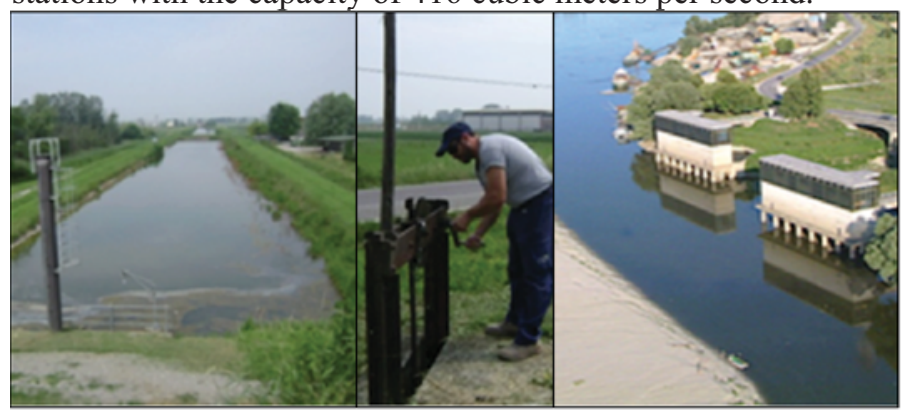

Fig. 3. Water distribution management pilot: open earth canal

(left), manual operation (center) and water intake (right)

The supply and irrigation network consists of open channels on earth. Relevant widths characterize the main canals and their filling for the irrigation season involves the use of substantial water volumes that are not always recoverable for irrigation purposes. Water losses are due to evaporation and infiltration through canal banks and bottom, as well as to the management of the irrigation network that requires the filing of long canals stretches and several minor streams to accommodate farmer needs. The irrigation network also acts as drainage network for the cultivated areas. The SWAMP project aims at enhancing the overall system efficiency by acting at farmers and irrigation consortium levels. At the farmer level, SWAMP provides a better estimation of water 
needs regarding both amounts of water and time of delivery. This precise estimation can be achieved by the implementation of the IoT infrastructure that enables the integration of groundbased information with the weather forecast. At the consortium level, the management of the irrigation network can benefit from the optimization of multiple water requests ensured by the technological platform. The platform collects water needs adjusting the operational management of canals by merging detailed information of water needs, weather forecasts, and multiple requirements from farmers served by the same irrigation infrastructure. In particular, the SWAMP project enables the monitoring, automation, and remote control of the major hydraulic infrastructures, through which CBEC manages the water distribution within the irrigation district. The renovation of the hydraulic infrastructures together with the implementation of the IoT platform and data analytics scheme is expected to enable a consistent enhancement of the water distribution management that will persist after the project life.

\section{B. Pilot: Smart precision irrigation (Spain - Intercrop)}

This pilot is located in Cartagena, Spain, in the premises of Intercrop Iberica and addresses several challenges of smart irrigation and water management of geographically distributed fields, aiming at demonstrating the use of IoT sensor platforms and drones to achieve increased crop yield with optimal water usage with minimum labor effort. Even though Cartagena is located on the coast, that is a dry area with a short rain season with intense rains in just a few days. A considerable amount of water comes from a desalination plant, what contributes to make it a scarce and expensive good. Farms are distributed over areas of roughly $30 \mathrm{~km}$ radius in intensive cropping area.

Pilot site is shown in Fig. 4. The immediate closeness of the control fields gives ideal circumstances to verify the effectiveness of the deployed methods. There are three growth periods during the project giving an opportunity to do iterative piloting and compare the results of different iterations.

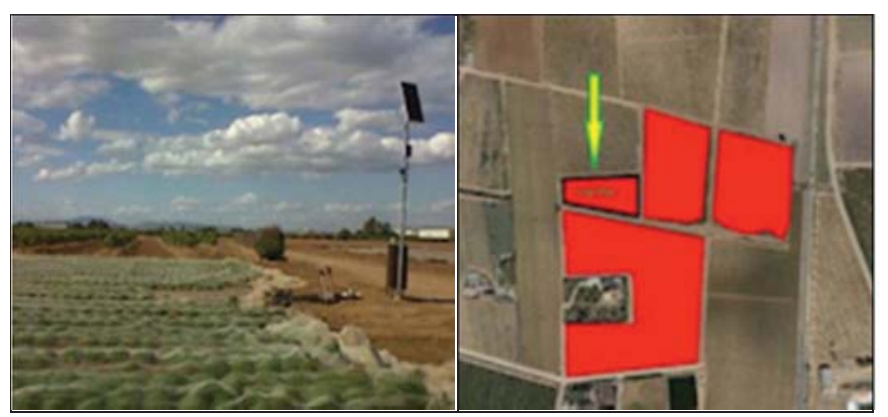

Fig. 4. Intercrop irrigation pilot site. In the right, the arrow shows the precise location of the pilot site

Growing period starts in early September and finishes by late April. Crops are from different ranges such as lettuce, endive, spinach, herbs, and bay leaf. These farms are irrigated separately through their proper reservoirs and irrigation systems. To demonstrate the effectiveness of the methods, we selected the pilot site from the same area with control field that is using traditional irrigation and monitoring methods. Irrigation system on the pilot field is managed using solenoid valves, and total water consumption can be monitored via water meters. Facilities used in piloting are a local weather station, online weather services, long life smart soil sensors, drone gateway, and real-time cloud data analysis.

\section{Pilot: Variable Rate Irrigation (Brazil)}

The MATOPIBA region encompasses the Brazilian states of Maranhão (MA), Tocantins (TO), Piauí (PI) and Bahia (BA), and is one of the most critical irrigated agricultural frontier in the country, located in the cerrado, a savannah climate subtype. Irrigation is mostly performed by thousands of center pivots, each one with an average size of 100 ha. The municipality of Luís Eduardo Magalhães (Fig. 5), the location for the center pivot pilot, is considered the capital of Brazilian agribusiness.

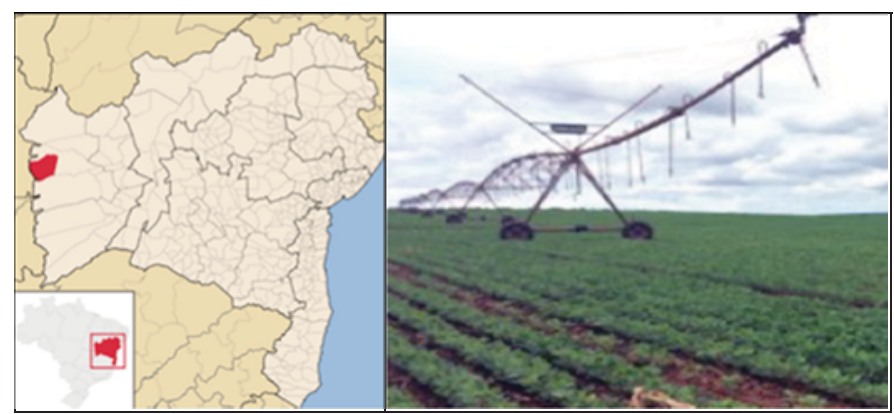

Fig. 5. Center-pivot irrigation pilot: location of the pilot site

(left) and center pivot irrigation for soybean crop (right)

The soybean production reached about 5.5 million tons in 2016. Despite such production, the farmers estimated $40 \%$ crop losses due to the 2015/16 season drought. The production from many properties was only 30 sacks per hectare, while an average of 58 sacks was expected. With irrigation and investments in soil fertility, some producers expect that this number can reach 80 sacks. Although irrigation is an alternative, its expansion depends on technologies that improve operating costs. For example, in 2015/16 the government imposed double charges to the energy used in irrigation, which costs seven sacks per hectare against three and a half sacks with a typical energy charging.

The main MATOPIBA pilot goal is to implement and evaluate a smart irrigation system based on Variable Rate Irrigation (VRI) for center pivots in soybean production. However, alternate crops like corn and cotton are also usual, including during the same season. Conventional center pivot irrigation systems help to provide a reliable supply of water, increase yields, and provide double cropping potential. The proposed smart irrigation will help the crop in the same way while making rational use of water and energy.

\section{Pilot: Precision Drop Irrigation (Brazil - Guaspari)}

One of the significant innovations of winemaking in State of São Paulo, Brazil, is occurring in the municipality of Espírito Santo do Pinhal in the Guaspari Winery. It is the transfer of wine grape harvesting to the winter season (June-August), when the thermal amplitude, insolation, and absence of rainfall are better for harvesting, similar to the great wine regions of the world. The different terroirs that compose the 
vineyard are divided into plots, which are harvested separately. In that growing area, altitude ranges between 1,000 $\mathrm{m}$ and 1,300 $\mathrm{m}$; nights are cold and optimal sunshine during the day provides an air temperature range between $10^{\circ} \mathrm{C}$ and $12^{\circ} \mathrm{C}$ at harvesting time, similar to that of large European regions. The soil is developed from granite rock, with good drainage, which is suitable for grapes destined to produce high-quality wines.

The application of water by an irrigation system depends on the crop, weather, and soil conditions. Even with the availability of several criteria and procedures for the performance of irrigation management, the technique has a low adoption rate by the farmers. Occasionally irrigation is based on only the experience of the grower, on the visualization of the wet soil surface or even on the perception of visual symptoms of plant water deficit, which, when identified late, can affect yield and product quality. The lack of knowledge about irrigation management procedures can lead to the failure of this water application practice, even if there is a system designed for a specific cropping area.

Guaspari Winery uses drip irrigation in its vineyards, with a single hose and emitters spaced at $0.5 \mathrm{~m}$, and measured the flow of $1.9 \mathrm{~L} \mathrm{~h}-1$. The experimental area has 1.1 ha and is shown in Fig. 6. The objectives of the Guaspari Winery Pilot are: a) to perform automatic measuring of soil water content at different soil depths and in several vineyards inside the winery's growing area; b) to store data in the cloud about soil water content be jointly processed with weather and crop information; c) To provide quick and accurate irrigation management information.

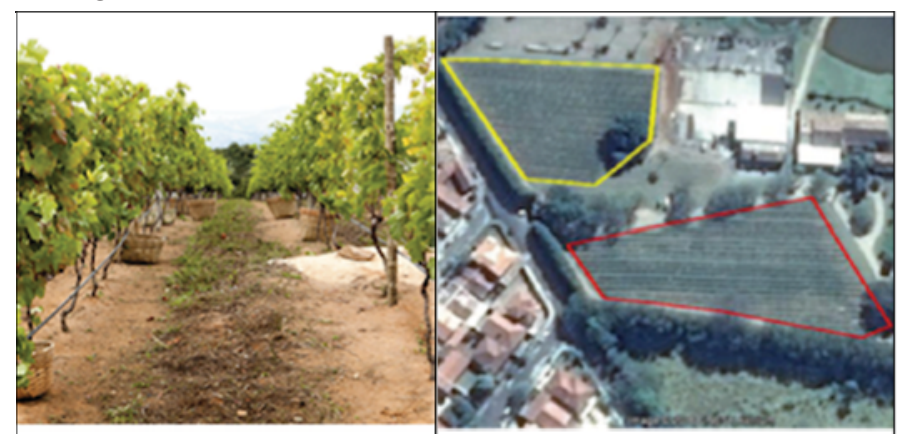

Fig. 6. Guaspari Winery: Vineyard and pilot location (right)

\section{SWAMP SCENARIOS}

The SWAMP Platform is being built on existing and new concepts and technologies, implementing the SWAMP vision (Fig. 1) through the SWAMP Architecture (Fig. 2), which may be flexible enough to deal with the needs of four different IoT pilots for smart water management in precision irrigation located in three countries. For making this vision to come true, a scenario-based architecture design and requirement gathering are being conducted to match the needs of crops and farmers' expertise with existing technological solutions.

Fig. 7 depicts the scenario representing the center-pivot with variable-rate irrigation pilot to be deployed in the MATOPIBA region in Brazil. The scenario captures both the farm and SWAMP Platform viewpoints and represents a future vision and not the current situation. A center pivot irrigates a circular agricultural plot of 100 hectares that alternates soybeans and cotton. The plot is further divided into different management zones, identified before the crop season and based on differences in the soil properties. Even though the crop is the same throughout the plot, each management zone receives a different irrigation prescription, to make available the same amount of water for plants regardless the way water is percolated and infiltrated in different soil types.

Two general types of sensors collect data for the SWAMP system: a) stationary sensors within the soil at the root system depth that continually measure metrics such as temperature and moisture, and; b) drones acting as flying sensors equipped with thermal/multispectral cameras or working as data mules by collecting sensor data and transmitting to the farm's office. The center pivot is electrified to make it able to control the variable rate irrigation sprinklers (actuators) and thus it can also be equipped with processing and communication capabilities. No special energy harvesting is predicted for the sensing devices. We will develop multiparametric probes for soil sensing, which include moisture, temperature and electrical conductivity sensors at three depths from the soil surface. In intensive agriculture areas the plan is also to experiment ultra low power wireless sensor solutions based on wake up radios combined with LoRaWAN [3]. Sensors will stay on standby and wake up for a few milliseconds. Sampling and transmission rates will be adjusted from a few minutes up to hours, according to the time of the day and the application requirements. This way, the probe sensing electronics combined with ZigBee or low power LoRaWAN will be powered by extended lifespan batteries. We consider using both ZigBee and LoRaWAN, for short and long distances, respectively. Other sensing devices deployed in low quantity or devices requiring heavier duty cycles, like a water source level sensor and weather station, will be solar powered.

Both the center pivot and drones are considered Field Fog Nodes (FFN) in the SWAMP Architecture. Notably, the FFN at the center pivot acts as a sensor aggregation point and as a distributor of actuator commands received from the SWAMP system. FFNs communicate with the nearest fixed attachment point of the SWAMP Platform, which usually is an onpremises Fog Hub Node (or just fog hub) located in the farm's office. Please notice that this choice aims at providing autonomous processing capacity to the farm at our pilot since it is in a region where Internet connections may suffer periods of instability. SWAMP allows FFNs to communicate directly with the Platform running in the cloud, whenever this is the preferred deployment choice. The FFNs send data directly to the SWAMP Platform located in the CFN at the farm's offices via different wireless technologies. In Fig. 7 it is represented by a Base Station, such as a WiFi access point, a LoRaWAN gateway, a ZigBee router or a Cell Base Station.

For this scenario, the functions performed by the SWAMP Architecture are divided up into local Fog and remote Cloud components. Heavy processing, such as irrigation models and analytics using smart algorithms (i.e., machine learning), is performed in the cloud. In our pilot, we will first experiment with existing irrigation models for precision agriculture where 
analytics plays the role of automatic parameter tuning. After enough data is obtained, analytical models will be tested for generating the irrigation prescription maps. External information is fed to the platform, such as crop yield models, meteorological data and historical data.

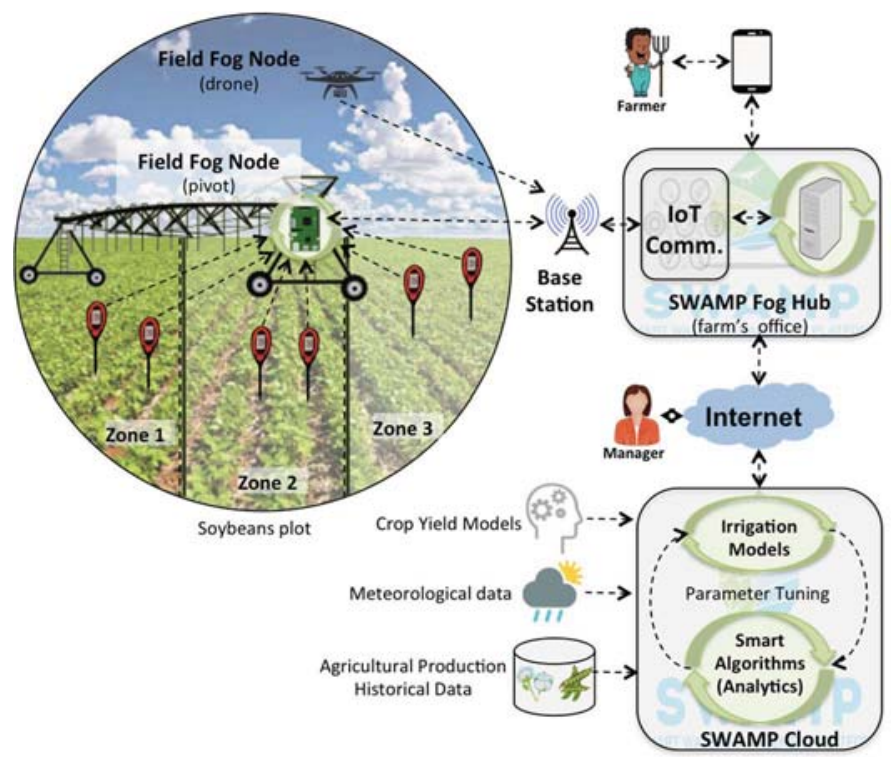

Fig. 7. Scenario representing the center-pivot irrigation pilot

Fig. 8 depicts the CBEC SWAMP pilot, focused on water distribution management. Water is conveyed from the Po River in Italy through a network of open canals automated by a set of pumps, valves, gauges and gates operated by CBEC.

The SWAMP platform estimates the water needs of individual farms by analyzing sensor data and other relevant information, generates the irrigation prescription maps and sends them to the farmers, who perform the irrigation using the method they see fit. The water need estimate for each farm is in turn fed into a component devoted to optimizing the management of water distribution, by sending commands to actuators installed in devices that control the water flow through the canals, up to the point farmers can use them. The purpose is to make more efficient use of the water deviated from the river. Fig. 8 does not explicitly show how the data is sent from sensors to the SWAMP platform because different technologies are used by farmers to transmit the data through the Internet. The SWAMP Platform is represented as a single component, whereas in practice a distributed cloud/fog architecture can be used, such as the one shown in Fig. 7.

\section{CONCLUSION}

IoT is the natural choice for smart water management applications, but its appropriateness is still to be proven in real settings with the deployment of on-site pilots. In this paper, we presented the SWAMP project, which develops IoT based methods for smart water management in precision irrigation, and pilots them in Italy, Spain, and Brazil. We introduced the SWAMP view, architecture, pilots and the scenario-based development process adopted in the project. SWAMP is still in its beginning but has already attracted the attention of farmers, agribusiness companies, and government agencies around the world. In its current stage, we visited and surveyed the pilots and explored the implications on the platform of different pilot sites, crops and requirements. The key insights so far refer to the commonalities and variability of features of pilots that can positively contribute to the design of a genuinely replicable SWAMP platform.

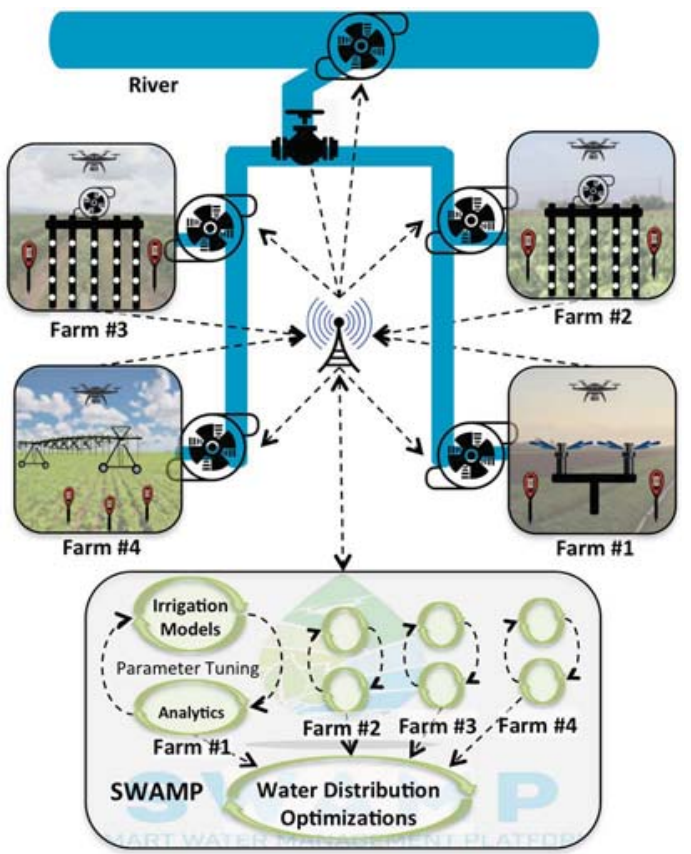

Fig. 8. Scenario representing the water distribution pilot

\section{REFERENCES}

[1] Atzori, L., Iera, A., Morabito, G., "The Internet of Things: A survey," Computer Networks, 54(15), October 2010.

[2] Bassi, A. et al., "Enabling Things to Talk: Designing IoT solutions with the IoT Architectural Reference Model," Springer, 2013.

[3] Bedogni, L. et al., "Dual-Mode Wake-Up Nodes for IoT Monitoring Applications: Measurements and Algorithms", IEEE ICC 2018, May 2018.

[4] Bonomi, et al., "Fog computing: A platform for Internet of Things and analytics," Book Chapter, Springer, 2014.

[5] Brewster, C. et al., "IoT in Agriculture: Designing a EuropeWide Large-Scale Pilot," IEEE Comm. Mag., September 2017.

[6] Doron, L., "Flexible and Precise Irrigation Platform to Improve Farm Scale Water Productivity," Impact, 2017(1), January 2017.

[7] FAO, "AQUASTAT: Water Uses," http://www.fao.org/nr/water/ aquastat/water_use, 2016, Accessed February 2018.

[8] Kamienski, C. et al., "Application Development for the Internet of Things: A Context-Aware Mixed Criticality Systems Development Platform," Computer Communications, May 2017.

[9] Kamilaris, A. et al., "Agri-IoT: A semantic framework for Internet of Things-enabled smart farming applications," IEEE WF-IoT 2016, December 2016.

[10] López-Riquelme, J. A., "A software architecture based on FIWARE cloud for Precision Agriculture," Agricultural Water Management, March 2017.

[11] Popović, T. et al., "Architecting an IoT-enabled platform for precision agriculture and ecological monitoring: A case study," Computers and Electronics in Agriculture, August 2017.

[12] Ramparany. F., et al., "Handling smart environment devices, data and services at the semantic level with the FI-WARE core platform," IEEE Intl. Conference on Big Data, October 2014.

[13] Weyrich, M., Ebert, C., "Reference Architectures for the Internet of Things," IEEE Software, 33(1), January 2016. 